\title{
Estilos de enseñanza en la educación física por subniveles de educación bajo la modalidad virtual
}

Teaching styles in physical education by education sublevels under the virtual modality

1 Christian Mauricio Sánchez Cañizares (iD) https://orcid.org/0000-0002-0677-7246 Universidad Técnica de Ambato, Carrera de Pedagogía de la Actividad Física y Deporte, Tungurahua, Ecuador cm.sanchezc@uta.edu.ec

2 Erick Gonzalo Gavilanes López (iD) https://orcid.org/0000-0003-1300-3430 Universidad Técnica de Ambato, Carrera de Pedagogía de la Actividad Física y Deporte, Tungurahua, Ecuador. gavilanes_erik@hotmail.com

3 Lenin Esteban Loaiza Dávila (iD) https://orcid.org/0000-0002-5769-2795 Universidad Técnica de Ambato, Carrera de Pedagogía de la Actividad Física y Deporte, Tungurahua, Ecuador.

e.loaiza@uta.edu.ec

4 Washington Ernesto Castro Acosta (iD) https://orcid.org/0000-0001-5028-9680 Universidad Técnica de Ambato, Carrera de Pedagogía de la Actividad Física y Deporte, Tungurahua, Ecuador. we.castro@uta.edu.ec

Artículo de Investigación Científica y Tecnológica

Enviado: 24/12/2021

Revisado: 29/12/2021

Aceptado: 12/01/2022

Publicado:08/03/2023

DOI: $\underline{\text { https://doi.org/10.33262/concienciadigital.v6i1.4.2028 }}$

Cítese Sánchez Cañizares, C. M., Gavilanes López, E. G., Loaiza Dávila, L. E., \& Castro Acosta, W. E. (2023). Estilos de enseñanza en la educación física por subniveles de educación bajo la modalidad virtual. ConcienciaDigital, 6(1.4), 781-798. https://doi.org/10.33262/concienciadigital.v6i1.4.2028

CONCIENCIA DIGITAL, es una Revista Multidisciplinar, Trimestral, que se publicará en soporte electrónico tiene como misión contribuir a la formación de profesionales competentes con visión humanística y crítica que sean capaces de exponer sus resultados investigativos y científicos en la misma medida que se promueva mediante su intervención cambios positivos en la sociedad. https://concienciadigital.org

La revista es editada por la Editorial Ciencia Digital (Editorial de prestigio registrada en la Cámara Ecuatoriana de Libro con No de Afiliación 663) www.celibro.org.ec 


\section{Palabras} claves: educación virtual, educación física, estilos de enseñanza, pandemia.

Keywords:

Physical

Virtual

education, Physical

Education,

Teaching

styles, pandemic.
Resumen

Introducción: La pandemia del COVID-19 cambió la manera en cómo se desarrollaba la educación, razón por la cual obligó a implementar la modalidad virtual de enseñanza de las ciencias, sin dejar de ser una excepción la Educación Física en donde los docentes se vieron en la necesidad de implementar diferentes estilos de enseñanza que permitieron desarrollar la nueva modalidad aplicada tomando en cuenta el subnivel de educación y otros factores. Objetivo: Determinar la aplicación de los estilos de enseñanza más utilizados en la Educación Física bajo la modalidad de educación virtual y su relación con el subnivel de educación impartido. Metodología: estudio propuesto con un enfoque de carácter cuantitativo, de tipo no experimental de alcance correlacional con un corte transversal. La muestra de estudio estuvo representada por 115 docentes de diferentes unidades educativas de la provincia de Tungurahua - ecuador. Como instrumento se aplicó el cuestionario DEMEVI validado estadísticamente y por criterio de expertos. Resultados. Se pudo determinar que los estilos más utilizados por parte de los docentes fueron el creativo y el tradicional con los cuales se imparten los conocimientos a los estudiantes y que existe una relación directa entre en subnivel de educación y la preferencia de estilo de enseñanza en un nivel estadístico de $\mathrm{P}<0.05$. Conclusión. Los estilos de aprendizaje con mayor preferencia en su aplicación por parte de los docentes fueron el estilo creativo y tradicional tomando en cuenta el subnivel de educación impartido, por lo que se puede deducir que se realiza una hibridación de estilos de enseñanza ya que estos se asemejan según los resultados obtenidos.

\section{Abstract}

Introduction. The COVID-19 pandemic changed the way education was developed, which is why it forced the implementation of the virtual modality of science teaching, without ceasing to be an exception Physical Education where teachers found themselves in the need to implement different teaching styles that allowed the development of the new modality applied considering the sub-level of education and other factors. Objective. To determine the application of the most used teaching styles in Physical Education under the virtual education modality and its relationship with the sub-level of education given. Methodology. Proposed study with a 
quantitative, non-experimental approach of correlational scope with a cross-sectional cut. The study sample was represented by 115 teachers from different educational units in the province of Tungurahua - Ecuador. The DEMEVI questionnaire was used as an instrument, validated statistically and by expert criteria. Results. It was possible to determine that the most used styles by teachers were the creative and the traditional with which knowledge is imparted to students and that there is a direct relationship between the sublevel of education and the preference of teaching style at a statistical level of $\mathrm{P}<0.05$. Conclusion. The learning styles with the greatest preference in their application by the teachers were the creative and traditional style considering the sub-level of education given, so it can be deduced that there is a hybridization of teaching styles since these are similar according to the results obtained.

\section{Introducción}

La enseñanza de la Educación Física es un arte en la cual se utiliza un conjunto de metodología, didáctica, estrategia y elaboración de materiales, por lo cual el docente debe manejar un repertorio de conocimientos sobre estas áreas para aplicar cada una de ellas en el momento propicio, al referirnos sobre los estilos de enseñanza es la forma de estructurar una clase que posibilite la formación integral de los dicentes.

Revisando la literatura hace referencia Mendoza y Gallardo (2016) el profesor no sólo enseña conocimientos, ayuda a los alumnos a "aprender a aprender" de manera autónoma y promueve su desarrollo cognitivo y personal mediante actividades críticas y explicativas que tengan en cuenta sus características y les exijan un procesamiento activo e interdisciplinario de la información para que construyan su propio conocimiento y no se limiten a realizar una simple recepción pasiva-memorización de la información, perfeccionado las facultades intelectuales y morales del alumno por medio de preceptos, ejercicios, ejemplos, etc.

La pandemia COVID-19 tuvo un impacto fuerte en el área de la educación física, los docentes no estaban preparados para realizar una enseñanza virtual, la educación física se lo desarrolla en espacios al aire libre siendo estos propicios para la práctica de la actividad física, recreación, ocio y uso adecuado del tiempo libre, sin embargo esto no fue un impedimento para poder impartir los conocimientos tanto teóricos y más aún prácticos y aunque sin la experiencia se empleó estrategias con el objetivo de estructurar clases virtuales que beneficien a los estudiantes, buscando como resultado un aprendizaje significativo, tanto docentes como estudiantes se adaptaron a esta modalidad en el cual 
implicaba el adecuado manejo de los recursos tecnológicos y metodológicos adaptando los estilos de enseñanza para llegar con el conocimiento a los estudiantes.

Al respecto Pavlovic (2021) en su estudio enfatiza sobre el impacto negativo por las prolongadas vacaciones escolares sobre la salud de los jóvenes, es fundamental comprender cómo la escolarización remota actual afecta la actividad física en los niños. Muchas escuelas y distritos escolares se enfrentan al desafío de encontrar los mejores enfoques para educación tangencial, incluida la Educación Física (EF).

La adaptación a la virtualidad en las diferentes asignaturas debido a la emergencia fue rápida, en la área la Educación Física tuvo un declive ya que este es práctica, en la que se evidenció un retroceso por la falta de manejo de los recursos informáticos o también llamados TIC's, siendo un problema fundamental el confinamiento donde se reducía los espacios de trabajo necesarios para la clase de (EF) dificultando su ejecución, respecto Pavlovic (2021) en consecuencia a la emergencia sanitaria dieron paso al distanciamiento social, la restricción de movilidad, perdida de hábitos saludables, causando que un gran porcentaje de la población se encuentre en el sedentarismo. Los expertos enfatizan la importancia de un estilo de vida físicamente activo para la salud y el manejo del estrés, con las medidas de bioseguridad necesarias.

En el presente estudio nos centramos en los estilos de enseñanza que más utilizan los docentes en la Educación Física modalidad virtual para impartir sus conocimientos ya que al ser una asignatura de componente práctico y necesitar de un espacio amplio y materiales para poder ejecutar las actividades se vio limitado al espacio reducido en casa tanto por el docente como por el estudiante, con el propósito de incentivar la creatividad e incluso darle una segunda oportunidad a los materiales con los que se cuenta en casa para aprovechar el tiempo y el espacio e incluso capacitarse en cómo utilizar las TIC's, implementando nuevas metodologías de enseñanza y adaptar los estilos de aprendizaje según las necesidades de los estudiantes para brindar un aprendizaje significativo tal y como hace referencia Garcés-Cobos et al. (2018) en su cita, dicho de otra manera, la trascendencia se da en el momento que el docente planifica tareas y actividades, para que el estudiante sea capaz de desarrollar habilidades y destrezas de aprendizaje propias, para luego ser replicadas en diferentes situaciones, Por ejemplo, facilitar el sentido de competencias (conocimientos), regulación de la conducta y control del comportamiento, desarrollar el sentido de compartir.

Los estilos de enseñanza es una forma de impartir conocimientos mediante una relación comunicativa-educativa que incluso esta depende del comportamiento del docente, su empatía y calidad humana; para poder efectivizar esta comunicación con sus estudiantes debe manejar un vocabulario simple, de fácil comprensión además de lo demostrativo por parte del docente y dependiendo del entorno en el cual se trabaja establecer la utilización de los distintos métodos y/o estilos de aprendizaje en función de los estudiantes, los 
objetivos de la clase, los contenidos del área y de esta manera conseguir un desarrollo cognitivo, psicológico y motriz de los dicentes.

La utilización diferentes estilos de aprendizaje es importante para evitar la rutina cuando se utiliza una sola de ellas, como hace referencia Castaño (2016), las estrategias que se usen con cualquier grupo deben variar tan frecuentemente como sea posible, evitando con ello la monotonía que genera la repetición excesiva de las mismas técnicas o procesos.

Según Mendoza y Gallardo (2016), al respecto hay que señalar que, la docencia no comienza y termina en "el aula", es decir, en la acción directa con los alumnos, implica decidir cómo diseñar los objetivos didácticos, seleccionar, ordenar, estructurar y temporalizar los contenidos, etc. (decisiones proactivas), cómo poner en práctica la programación y comprobar sus resultados en el aula para introducir, si es preciso, cambios en el desarrollo de las clases con el fin de alcanzar los objetivos que se ha propuesto (decisiones interactivas) y cómo evaluar, una vez dado el programa establecido, la acción nuestra como docentes para realizar las modificaciones necesarias en la intervención y programación futuras (decisiones postactivas).

Los estilos tradicionales son aquellos que por lo general utilizan un mando directo y es el docente quien se encarga de impartir los conocimientos limitando la participación de los estudiantes, así hace referencia Mendoza y Gallardo (2016), la enseñanza masiva centrada en el profesor y basada en la instrucción directa siendo la comunicación unidireccional. El profesor ordena sin dar posibilidad a la individualización ya que prescribe la tarea a realizar por todos y de la misma manera, con los mismos requerimientos.

Estilos que posibilitan la participación el docente permite la participación activa del estudiante en la clase, inclusive le hace partícipe en la toma de decisiones dentro de los contenidos y planteamiento de actividades acordes a los objetivos de la clase, el docente permite que el trabajo se realice entre estudiantes (parejas o grupos) en donde uno de los estudiantes realiza el rol de docente siempre con la guía del profesor e incluso puede utilizar una ficha de observación en donde el estudiante anote los aspectos a tener en cuenta en la clase, al respecto Mendoza y Gallardo (2016) enseñanza basada en el principio de actividad. Los alumnos participan activamente en su aprendizaje y en el de sus compañeros de clase.

El papel del docente es realizar preguntas que guíen al estudiante a la ejecución de las actividades llamado también descubrimiento guiado o a su vez plantea problemas que motiven al descubrimiento de nuevas maneras de ejecución motriz, así lo plantea Mendoza y Gallardo (2016) enseñanza basada en el desarrollo de las capacidades de los alumnos para que realicen aprendizajes significativos por sí solos (aprender a aprender). El profesor indica la tarea que hay que realizar, pero no cómo hay que realizarla, la forma de abordarla. Se solicita a los estudiantes que aprovechen su propio feedback. 
Así hace referencia Mendoza y Gallardo (2016) enseñanza basada en el desarrollo potencial creador de los alumnos. El profesor actúa como facilitador del aprendizaje y de la producción creativa, dinamizador del grupo o grupos de trabajo. Las situaciones de enseñanza que plantea permiten a los alumnos preguntar, examinar, indagar, resolver problemas de forma original, etc.

Es importante que cada estilo aquí descrito irá de acuerdo con el criterio del docente quien según su experiencia aplicará uno de ellos o dependiendo de la circunstancia usará varios en una misma sesión, lo importante es la diversidad y evitar la monotonía como ya se habló anteriormente.

La educación física virtual se introdujo en nuestro medio debido a la pandemia que vive el mundo a causa del COVID-19, que pese al esfuerzo que realizaron las autoridades por contrarrestar o frenar la propagación del virus no se lo consiguió, sin embargo a la actualidad y ya con las vacunas se está tratando de volver a la normalidad, para ello cito a Zambrano (2021), quien en su estudio hace referencia al respecto, La pandemia declarada por la Organización Mundial de la Salud (OMS) la segunda semana del mes de marzo 2020 por causa del COVID-19, acarreó consecuencias en las actividades habituales que desempeña el ser humano en todos los ámbitos: socioeconómicos, educativos, laborales, sociales, familiares y religiosos. En ese sentido, las instancias gubernamentales en todo el mundo adoptaron medidas drásticas para evitar la propagación del virus y tratar de contrarrestar los decesos causados por la enfermedad (Sánchez et al., 2020).

Aunque la pandemia ha sido causa de muchos despidos y bajas de sueldos lo que ha desmotivado a las personas para realizar activamente sus actividades en el ámbito personal y profesional debido a las malas decisiones que ha tomado el gobierno, en nuestra área nos ha tocado buscar alternativas que posibiliten la participación activa de los docentes y los estudiantes por lo que el Ministerio de Educación implementó un plan educativo "Aprendemos juntos en casa" en donde se da prioridad al currículo mediante el trabajo por proyectos planteados por los docentes.

En este aspecto Zambrano (2021), en su estudio hace referencia Frente a todas las adversidades suscitadas por la emergencia sanitaria, la educación inicial, primaria y secundaria no podía detenerse. Bajo esta incertidumbre, el Ministerio de Educación del Ecuador implementó el plan educativo 'Aprendemos juntos en casa' en donde se priorizó el currículo para todas las regiones. Este método dispone de recursos educativos digitales, canales de televisión, programas en radio, portales, plataformas virtuales y lineamientos para estudiantes, docentes, padres de familia y autoridades con la finalidad de dar continuidad al proceso enseñanza-aprendizaje desde los hogares mediante el apoyo de la Tecnología de la Información y Comunicación (TIC). 
Pese a la implementación del plan educativo la falencia por parte de los docentes en el ámbito de la utilización de las TIC's limitó en un inicio la impartición de conocimientos y de cómo aplicar o utilizar los estilos de enseñanza, metodología, estrategias educativas, didáctica, utilización de material, etc., por lo que a más de empezar a ser un obstáculo también motivó a los docentes a capacitarse en el uso de herramientas tecnológicas pedagógicas para de esta manera atender las necesidades de los estudiantes al respecto (Ossandón et al., 2021). La didáctica de la Educación Física se tuvo que adaptar a aquel contexto y también los estilos de enseñanza.

Por ello es necesario acotar que según lo citado en su estudio por Bou-Sospedra et al. (2020), el estilo de enseñanza hace referencia al cómputo de herramientas metodológicas empleadas por el docente para transmitir aptitudes y conocimientos al alumno de una manera eficaz, motivante y duradera (Fernández \& Ladrón de Guevara, 2015). Existe una gran variedad de evidencia científica en lo que respecta a estilos de enseñanza y estilos de aprendizaje, abriendo un amplio abanico de posibilidades y matices, por lo que es importante que el docente conozca el estilo de aprendizaje de cada estudiante, y concretamente de cada grupo, para desarrollar un aprendizaje significativo...

A ello hay que acotar la importancia del uso de las distintas plataformas existentes en el medio informático por lo que los docentes de Educación Física adaptaron los contenidos de cómo impartir sus conocimientos a través de aplicaciones como Zoom y Google Meets, el uso de plataformas Moodle (las más utilizadas por el uso de video y para entrega de tareas), así como también del uso de plataformas digitales y tratar de emular las clases como si fueran de manera presencial, por lo que según lo citado por Ruiz y Dávila (2016), es importante destacar que el simple hecho de usar las TIC en la educación universitaria no produce necesariamente cambios significativos en la calidad del tipo de formación que se desarrolla en las instituciones académicas. El impacto de las TIC sobre la educación depende más bien del contexto de uso y de la finalidad con que son utilizadas y de la aplicación efectiva que de ellas hacen los profesores y alumnos en los planteles educativos y en las aulas; son estos factores los que finalmente determinan el mayor o menor impacto en las prácticas educativas y su mayor o menor capacidad para transformar la enseñanza y mejorar el aprendizaje.

De esta manera y adaptando los estilos de enseñanza a esta realidad que actualmente estamos viviendo a través de la asignación de tareas y resolución de problemas en la construcción de su propio conocimiento por parte de los estudiantes, quienes se han visto más involucrados en la Educación Física no solo por la realización de actividades físicomotrices sino por la creación de materiales para el uso en el aula a partir de material reciclado, aplicando un mando directo, mando directo modificado y asignación de tareas que se encuentran o pertenecen al estilo tradicional que de igual manera permite el desarrollo de los estudiantes. 
Como manifiesta Ossandón et al. (2021) en su estudio continuando con la evolución histórica de los Estilos de Enseñanza, corresponde mencionar una corriente española que ha contribuido en la materia. Estilos propuestos por Muska Mosston, generó la siguiente clasificación: a) estilos tradicionales: mando directo, mando directo modificado uno de los estilos más utilizados es el tradicional con el cual el docente ha aplicado un mando directo y asignación de tareas promoviendo de esta manera una utilización correcta de este estilo, otro de los estilos más utilizados es el participativo que incentiva a los estudiantes a construir su conocimiento a partir de la propuesta de problemas por parte del docente en donde implica cognitivamente al alumno, este estilo y pese a estar en un trabajo virtual ha permitido que los estudiantes se motiven ya que se ven beneficiados en una enseñanza recíproca, es decir interactuar con sus compañeros adoptando el rol de docente para conseguir mejoras en la realización de las actividades a través de las experiencias intrínsecas y extrínsecas.

Según lo citado por Sánchez et al. (2020), estos estilos de enseñanza de la Educación Física se pueden argumentar con los trabajos de Aragón y Hernández que sostienen que los estilos más utilizados son la instrucción o mando directo, desde el punto de vista tradicional y desde el ámbito constructivo, es el cognitivo que abarca los estilos por descubrimiento y resolución de problemas. Sumando a ello la perspectiva motivacional que contribuye al aprendizaje significativo (Ruiz \& Dávila, 2016).

Por ello la importancia de la utilización de herramientas tecnológicas que posibiliten la inclusión de todos los estudiantes en la Educación Física y aquí la importancia de la planificación por parte del docente en este ámbito en donde debe incluir el uso de plataformas digitales como material de apoyo en donde de manera sistemática se logre una excelente organización y prevalezca por sobre todo una buena comunicación y de esta manera promover el desarrollo de clases que tengan éxito sin olvidarnos de la evaluación que es parte fundamental de la educación, así hace referencia a Sánchez et al. (2020) de lo citado por él en el libro la didáctica de la Educación Física en Educación Infantil y Primaria en las propuestas metodológicas para una Educación Física del siglo XXI describe la importancia y recomienda herramientas digitales para planificar de forma sistemática donde sobresale la organización, la comunicación, el desarrollo de sesiones y la evaluación que llevaran al éxito el proceso educativo. La educación online es totalmente viable hacia una educación a distancia donde el estudiante puede acceder a video llamadas o reuniones virtuales, contenidos y tareas. Por lo que propone algunas herramientas y recursos digitales que pueden ser utilizados en las clases de Educación Física como; la herramienta 2.0, aprendizaje basado en juegos-gamificación, las aplicaciones móviles de actividad física en IOS/ANDROID y los blogs y recursos electrónicos. 
La tecnología ha tenido un gran avance y el acceso a la misma es muy fácil y en especial para nuestros estudiantes quienes con un teléfono celular tienen acceso a todo el entorno virtual a través de las aplicaciones y sus juegos, por lo tanto como docentes no estamos exentos del uso de la misma, hoy en día debemos verla como una oportunidad para llevar la educación física a otro nivel, muestra de ello es la adaptación de los estilos de enseñanza a los estilos de aprendizaje de nuestros estudiantes que hemos tenido que aplicar en esta modalidad virtual, si vemos el lado positivo de la tecnología es que nos permite estar en constante comunicación y eso para la educación física es fundamental y partiendo de allí podemos buscar una formación en nuestros estudiantes eficaz en los físico, psicológico, social, motriz, etc., en pro de mejorar su estilo y calidad de vida.

Por ello según lo citado por Fernández y Ladrón de Guevara (2015), en la actualidad, los móviles, los ordenadores portátiles, las tabletas, son una herramienta fundamental en la vida cotidiana de las personas. El ser humano tiende continuamente a la socialización, es decir, las personas buscan una conexión o unión a medios o instrumentos que promuevan las relaciones interpersonales y el intercambio de contenidos particulares.

Por lo tanto y según lo citado por González (2017) si en la clase de educación física se consigue que los estudiantes disfruten mientras adquieren un nivel básico de destreza motriz, es probable que continúen practicando en sus ratos libres. Sin embargo, si la clase de educación física se convierte en una experiencia traumática caracterizada por la ausencia de motivación y que pone de manifiesto la incompetencia motriz, será difícil que el niño o adolescente quiera comprometerse con una práctica física continuada .Dado que la literatura refleja claramente un efecto trans-contextual de que lo que ocurre en el aula va más allá del centro educativo influyendo en la vida del estudiante, resulta de sumo interés la correcta formación del docente de educación física en estrategias didácticas y motivacionales que ayuden a la promoción de la actividad física.

Y por otro lado según lo citado en su estudio Díaz (2020), para suscribir una definición de aprendizaje móvil que se adapte a la singularidad de la EF, la literatura señala dos grandes tradiciones: una de carácter tecno céntrica y otra de carácter tecno pedagógica .Desde este trabajo se conciben el Mobile Learning como «cualquier tipo de aprendizaje que tiene lugar en entornos y espacios de aprendizaje que tienen en cuenta la movilidad de la tecnología, la movilidad de los estudiantes y la movilidad del aprendizaje». El énfasis focalizado en la movilidad del alumnado, sus experiencias y los espacios de prácticas, más que en concepciones centradas en los propios dispositivos móviles, se ajusta a los objetivos de la EF. Así entendido, el Mobile Learning puede ser un valioso instrumento pedagógico para el aprender haciendo y reflexionando «en», «para»y «sobre» la acción, donde usuarios y usos tecnológicos se complementan. 


\section{Metodología}

Enfoque cuantitativo de tipo no experimental por alcance correlacional y de corte transversal, basado en la aplicación de los métodos analítico e hipotético deductivo para la fundamentación teórica y resolución de las hipótesis planteadas.

La muestra de estudio estuvo conformada por 115 docentes de diferentes unidades educativas de la provincia de Tungurahua - Ecuador, se considera un muestreo no probabilístico por voluntariado ya que se trabajó con aquellos docentes que sin ningún compromiso aceptaron su participación en el desarrollo de la investigación.

La técnica utilizada fue la encuesta y como instrumento el cuestionario DEMEVI adaptado para docentes que consta de 36 ítems de respuestas con escala de Likert, el cual fue validado por métodos estadísticos y criterio de expertos.

El procesamiento estadístico de los datos y resultados del trabajo de investigación se realizó a través del programa SPSS, versión 25 IBM para Windows, donde se utilizaron pruebas de estadística descriptiva para las variables cuantitativas y un análisis de frecuencia relativas (porcentajes) para las variables cualitativas que caracterizan la muestra de estudio. Para el análisis de significación general de la investigación, se aplicó en primera instancia una prueba de normalidad para muestras mayores a 50 datos como es la de Kolgomorov-Smirnov, que determinó la aplicación de la prueba no paramétrica U de Mann-Whitney para variables independientes de estudio y en el proceso final de relación entre el subnivel de educación y la preferencia de estilos de aprendizaje se aplicó la prueba Chi-cuadrado de Pearson para variables de carácter cualitativo.

\section{Resultados}

En primer lugar, se procedió a la caracterización de la muestra de estudio (tabla 1).

\section{Tabla 1}

Caracterización de la muestra de estudio

\begin{tabular}{|c|c|c|c|c|c|c|c|c|}
\hline \multirow{2}{*}{ Sexo } & \multirow{2}{*}{$\mathrm{f}$} & \multirow{2}{*}{$\%$} & \multicolumn{3}{|c|}{ Edad (años) } & \multicolumn{3}{|c|}{ Experiencia laboral (años) } \\
\hline & & & M & DS & $\mathrm{P}$ & M & DS & $\mathrm{P}$ \\
\hline Masculino & 69 & 60.0 & 40.65 & 8.57 & \multirow{3}{*}{$0.000 *$} & 10.96 & 8.37 & \multirow{3}{*}{$0.592 * *$} \\
\hline Femenino & 46 & 40.0 & 33.09 & 10.19 & & 10.37 & 8.22 & \\
\hline Total & 115 & 100.0 & 37.63 & 9.93 & & 10.72 & 8.28 & \\
\hline
\end{tabular}

Nota. Distribución de frecuencia (f) y porcentajes (\%) de las variables del sexo y valores medios (M) con desviaciones estándares (DS) de las variables de la edad y experiencia laboral, con diferencias significativas en un nivel de $\mathrm{P}<0.05(*)$ y $\mathrm{P} \geq 0,05(* *)$ entre los grupos por sexo.

La muestra presenta un mayor número de integrantes del sexo masculino, en base a la edad de los participantes se evidencio una diferencia de 7.56 años de edad media a favor 
de sexo masculino sobre el femenino, con existencia de diferencias significativas en un nivel de $\mathrm{P}<0.05$, en relación a la experiencia laboral la diferencia entre sexos es menor a la unidad, sin existencia de diferencias significativas con un valor de $\mathrm{P} \geq 0,05$, determinando en relación a la experiencia una igualdad por grupos de sexo.

Para el análisis fue necesario conocer la distribución de la muestra en relación con el subnivel de educación en el cual los docentes desarrollaban su ejercicio profesional (tabla 2).

\section{Tabla 2}

\section{Distribución muestral por subniveles de educación}

\begin{tabular}{ccc}
\hline Subnivel de educación & Frecuencia & Porcentaje \\
\hline Inicial & 21 & 18.3 \\
Básica elemental & 21 & 18.3 \\
Básica media & 25 & 21.7 \\
Básica superior & 23 & 20.0 \\
Bachillerato & 25 & 21.7 \\
\hline Total & 115 & 100.0 \\
\hline
\end{tabular}

La distribución por subniveles de educación determino una media de $23 \pm 2$ integrantes por cada uno, siendo los subniveles de educación básica media y bachillerato los que en un porcentaje mayor presentaron en sus grupos.

Se analizaron los resultados enfatizando la frecuencia y porcentaje de respuestas en base a las 5 opciones de respuesta en cada estilo de enseñanza, se realizó el cálculo de los puntajes obtenido por cada estilo, en relación con el número de ítems determinados en el instrumento y se categorizo cada estilo segmentándolos por el subnivel de educación en el cual el docente ejercía su labor profesional (tabla 3).

Tabla 3

Distribución de los estilos de enseñanza por subniveles de educación

\begin{tabular}{cccc}
\hline Subnivel de educación & Estilo de enseñanza & Frecuencia & Porcentaje \\
\hline \multirow{3}{*}{ Inicial } & Estilo tradicional & 5 & 23.8 \\
& Estilo socializador & 5 & 23.8 \\
& Estilo cognitivo & 5 & 23.8 \\
& Estilo creativo & 6 & 28.6 \\
\cline { 2 - 4 } & Total & 21 & 100.0 \\
\hline \multirow{2}{*}{ Básica elemental } & Estilo tradicional & 1 & 4.8 \\
& Estilo participativo & 6 & 28.6 \\
& Estilo socializador & 2 & 9.5 \\
& Estilo cognitivo & 5 & 23.8 \\
& Estilo creativo & 7 & 33.3 \\
\hline
\end{tabular}


Tabla 3

Distribución de los estilos de enseñanza por subniveles de educación (continuación)

\begin{tabular}{cccc}
\hline Subnivel de educación & Estilo de enseñanza & Frecuencia & Porcentaje \\
\hline \multirow{3}{*}{ Básica media } & Estilo tradicional & 12 & 48.0 \\
& Estilo participativo & 4 & 16.0 \\
& Estilo socializador & 1 & 4.0 \\
& Estilo cognitivo & 4 & 16.0 \\
& Estilo creativo & 4 & 16.0 \\
\cline { 2 - 4 } & Total & 25 & 100.0 \\
\hline \multirow{3}{*}{ Básica superior } & Estilo tradicional & 11 & 47.8 \\
& Estilo participativo & 2 & 8.7 \\
& Estilo socializador & 1 & 4.3 \\
& Estilo cognitivo & 1 & 4.3 \\
& Estilo creativo & 8 & 34.8 \\
\cline { 2 - 4 } & Total & 23 & 100.0 \\
\hline \multirow{2}{*}{ Bachillerato } & Estilo tradicional & 6 & 24.0 \\
& Estilo participativo & 6 & 24.0 \\
& Estilo socializador & 1 & 4.0 \\
& Estilo cognitivo & 4 & 16.0 \\
& Estilo creativo & 8 & 32.0 \\
\hline
\end{tabular}

En el subnivel de educación inicial y básico elemental el estilo de enseñanza más utilizado por los docentes es el creativo dado las características físico-psicológicas de los niños que pertenecen a este nivel de educación (4 - 5 años), que promueven el desarrollo integral del niño mediante la utilización del juego y actividades lúdicas que fomenten las capacidades físicas, motrices, cognitivas, sociales, etc.

En el subnivel de educación media y superior el estilo que más utilizan los docentes es el tradicional dado las características propias de los estudiantes por la edad en la que se encuentran (6 a 14 años) y que requieren de una orientación más directa del docente y permite una enseñanza sistemática de las acciones a ejecutar por parte del estudiante en el cual recae que responda de manera adecuada.

En el subnivel de educación bachillerato el estilo de enseñanza más utilizado por los docentes es el creativo ya que en esta edad los estudiantes necesitan fortalecer su conocimiento y crearlo a partir de sus habilidades y destrezas físicas y cognitivas.

En educación básica inicial, básico elemental y bachillerato el estilo que se utiliza con mayor frecuencia es el estilo creativo dado que en los niños y jóvenes de estos niveles promueve el interés por el desarrollo de su creatividad y su imaginación a través de la utilización del juego como herramienta metodológica y como es su naturaleza y parte de su característica fomenta el desarrollo físico, motriz, intelectual entre otros aspectos que le sirven al estudiante para fortalecer su calidad de vida y al docente construir el 
conocimiento mediante la resolución de problemas como hace referencia en lo citado en su estudio Rosa et al. (2018), indica que los estilos creativos surgen como métodos creativos de resolución de problemas, e incluye en este este grupo la lluvia de ideas corporal y la improvisación corporal.

Por otro lado según los resultados obtenidos en el presente estudio el estilo tradicional también se encuentra dentro de los más utilizado por los docentes en especial en los subniveles de educación media y superior ya que permite un mejor control de estos grupos, por una parte por la instrucción directa que reciben los estudiantes del docente y por otra porque dentro de este estilo tenemos variedad de oportunidades para la impartición de conocimientos como Mando Directo, Modificación del Mando Directo y Asignación de Tareas, que por la edad en la que se encuentran los estudiantes sirven sobre todo para mantener la disciplina y realizar las actividades que requieren de varias repeticiones para que sean bien ejecutadas.

Para entender de mejor manera la relación entre los subniveles de educación y las preferencias de estilos de enseñanza se estableció una tabla cruzada (tabla 4).

\section{Tabla 4}

Relación entre subniveles de educación y preferencia de estilos de enseñanza

\begin{tabular}{ccccccc}
\hline \multirow{2}{*}{$\begin{array}{c}\text { Subniveles de } \\
\text { educación }\end{array}$} & $\begin{array}{c}\text { Estilo } \\
\text { tradicional }\end{array}$ & $\begin{array}{c}\text { Estilo } \\
\text { participativo }\end{array}$ & $\begin{array}{c}\text { Estilo } \\
\text { socializador }\end{array}$ & $\begin{array}{c}\text { Estilo } \\
\text { cognitivo }\end{array}$ & $\begin{array}{c}\text { Estilo } \\
\text { creativo }\end{array}$ & Total \\
\hline $\begin{array}{c}\text { Inicial } \\
\text { Básica } \\
\text { elemental }\end{array}$ & 5 & 0 & 5 & 5 & 6 & 21 \\
$\begin{array}{c}\text { Básica media } \\
\text { Básica }\end{array}$ & 12 & 6 & 2 & 6 & 6 & 21 \\
$\begin{array}{c}\text { superior } \\
\text { Bachillerato }\end{array}$ & 11 & 4 & 1 & 4 & 4 & 25 \\
\hline Total & 35 & 6 & 1 & 1 & 8 & 23 \\
\hline
\end{tabular}

La relación entre estas 2 variables permitió determinar que, en el contexto estudiado los estilos de enseñanza más frecuentes es el tradicional y creativo, ya que depende de la formación y experiencia del docente frente a sus alumnos.

Como comprobación de hipótesis se aplicó un análisis estadístico para determinar si esta relación descriptiva presentada anteriormente tenía un sustento significativo a nivel estadístico (tabla 5). 
Tabla 5

Análisis estadístico de relación entre variables

\begin{tabular}{cccc}
\hline Estadísticos & Valor & df & $\begin{array}{c}\text { Significación } \\
\text { asintótica } \\
\text { (bilateral) }\end{array}$ \\
\hline Chi-cuadrado de Pearson & 30.798 & 16 & 0.014 \\
\hline N de casos válidos & \multicolumn{3}{c}{115} \\
\hline
\end{tabular}

En el proceso de comprobación estadística, se aplicó la prueba Chi-cuadrado de Pearson, la cual al 95\% (0.05) de confiabilidad y 16 grados de libertad presentó un valor crítico de 26.3, el cual es inferior al obtenido en la investigación (30.798), respaldado de una significación asintótica bilateral en un nivel de $\mathrm{P}<0.05$ que determina que existe una relación entre el subnivel de educación en el cual imparten clases los docentes estudiados y la preferencia de los estilos de aprendizaje presentados.

\section{Discusión de los resultados de la investigación}

Según el estudio realizado por Zapatero (2017) los resultados sugieren que los docentes utilizan de forma mayoritaria estilos tradicionales o centrados en el docente, lo que concuerda con el presente estudio en el cual en el subnivel de educación básica media y superior donde el docente utiliza un estilo tradicional en el cual desempeña el rol principal limitando a que los estudiantes simplemente sean los espectadores y ejecuten las actividades según lo establecido por el docente, esto dado las circunstancias actuales por la modalidad virtual.

En el presente estudio los estilos de aprendizaje que mayor incidencia tienen son los creativos. Los resultados demuestran una tendencia a los estilos de enseñanza Participativos. Teniendo en cuenta que el aprendizaje creativo promueve el desarrollo de la imaginación del estudiante dejándolo participar de una manera activa y reflexiva en la búsqueda de solución a los problemas planteados.

Ossandón et al. (2021) los resultados indican que los cinco estilos de enseñanza fueron mayoritariamente utilizados, destacando en el primer lugar el estilo de mando directo con lo que concuerda con el presente estudio en el cual el estilo tradicional es utilizado en el subnivel de educación básica media y superior dadas las características físicas, fisiológicas, comportamentales y psicológicas de los estudiantes que a su vez en los diferentes subniveles y según sus edades requieren de la utilización de un estilo tradicional.

Según el estudio de Pacheco y Maldonado (2017) los resultados mostraron que la mayoría de los estilos de aprendizaje se relacionan con las teorías de enseñanza, exceptuando el estilo reflexivo. El estilo de aprendizaje activo se relaciona con la teoría de enseñanza 
tradicional... que se relaciona con el estudio realizado en el que el estilo tradicional y creativo son los más utilizados por los docentes dado las características propias del área de Educación Física, sus contenidos, estructura, objetivos, resultados de aprendizaje y porque no de la evaluación que es aplicada en todo el proceso educativo.

En el estudio de Rosa et al. (2018) se hace necesario el desarrollo de modelos pedagógicos híbridos que permitan satisfacer las necesidades de los estudiantes en contextos concretos. Que en concordancia con el presente estudio se requiere una hibridación de los estilos de enseñanza dado la modalidad en la que los docentes están trabajando y la adaptación que deben hacer de los contenidos y objetivos de la Educación Física al trabajo virtual, para brindar las herramientas adecuadas en la formación integral del estudiante mediante el uso de plataformas digitales en todas sus estructuras, adaptándolas a esta modalidad virtual para que la educación se produzca de una manera efectiva ya que requiere de la presencia del docente y para ello en la modalidad en la que actualmente el docente trabaja le permite realizar clases sincrónicas y asincrónicas para fomentar el desarrollo adecuado de los estudiantes.

\section{Conclusiones}

- La evaluación realizada permitió determinar que según los diferentes subniveles de educación hay preferencia de estilos de enseñanza teniendo como prevalencia el estilo creativo para los subniveles inicial, básica elemental y bachillerato y el estilo tradicional en los subniveles básica media y básica superior.

- De igual manera se estableció si existe una relación entre el subnivel de educación y los estilos de enseñanza, estableciendo que descriptiva y estadísticamente existe una relación que permite establecer que el subnivel de educación tiene una dependencia directa con el estilo de enseñanza que el docente selecciona, respaldado en la edad de los educandos y en los procesos cognitivos, físicos y psicológicos que estos desarrollan.

- Al implementar los estilos de enseñanza por parte de los docentes estos deben buscar la manera de realizar una hibridación en los mismos para de esta manera trabajar de manera adecuada en la formación integral de los estudiantes dado que la modalidad virtual en la que se está trabajando con la utilización de las TIC's permite trabajar de manera sincrónica y asincrónica para que el aprendizaje y evaluación de los estudiantes sea adecuado según los subniveles de educación en el que se encuentren. 


\section{Referencias bibliográficas}

Bou-Sospedra, C., González-Serrano, M. \& Jiménez, M. (2020). Estudio de los estilos de enseñanza-aprendizaje desde la perspectiva de los tres agentes educativos: alumnos, docentes y familiares, 330,331.

Castaño Ruiz, J. (2016). Propuesta didáctica para el área de educación física: educación primaria. Wanceulen Editorial. 31.

Díaz Barahona, José. (2020). Retos y oportunidades de la tecnología móvil en la educación física, 763.

Fernández Espínola, C. \& Ladrón de Guevara, L. (2015). El uso de las TIC en la Educación Física actual (2015), 18.

Garcés-Cobos, L. F., Montaluisa-Vivas, Á. \& Salas-Jaramillo, E. (2018). El aprendizaje significativo y su relación con los estilos de aprendizaje, 234.

González Cutre, D. (2017). Estrategias didácticas y motivacionales en las clases de educación física desde la teoría de la autodeterminación, 45.

Mendoza Pérez, A. \& Gallardo Vázquez, P. (2016). Didáctica de la actividad física y deportiva: metodología, estilos de enseñanza, programación y evaluación. Wanceulen Editorial. https://elibro.net/es/lc/uta/titulos/63201, 29,34.

Ossandón Jiménez, E., Ramírez Silva, D., Torres Rojas, B., Santander Reveco, I., Uribe Uribe, N. \& Mujica Johnson, F. (2021). Estilos de enseñanza en Educación Física escolar en el contexto de pandemia, 46,48.

Pacheco Rios, R. \& Maldonado Maldonado, E. (2017). Relación entre los estilos de aprendizaje y las teorías de enseñanza

Pavlovic, B.M. (2021). Public Health Keeping children healthy during and after COVID19 pandemic: meeting youth physical activity needs, 2-3.

Rosa, A., García-Cantó, E. \& Pérez, J.J. (2018). Métodos de enseñanza en educación física: desde los estilos de enseñanza hasta los modelos pedagógicos, 10.

Ruiz Bolívar, C. \& Dávila Alirio, A. (2016). Propuesta de Buenas Prácticas de Educación Virtual en el Contexto Universitario, 3.

Sánchez Encalada, E. D., Ávila Mediavilla, C. M., García Herrera, D. G. \& Bravo Navarro, W. H. (2020). El proceso de enseñanza-aprendizaje de la Educación Física en época de pandemia, 458,459. 


\section{DDigital}

\section{ISSN: 2600-5859}

Vol. 6 No. 1.4, pp. 781 - 798, marzo 2023

www.concienciadigital.org

Zambrano Vacacela, L. L. (2021). Estilos de enseñanza de docentes en el Ecuador en contexto COVID-19, 137,138, 139.

Zapatero Ayuso, J. A. (2017). Beneficios de los estilos de enseñanza y las metodologías centradas en el alumno en educación física.

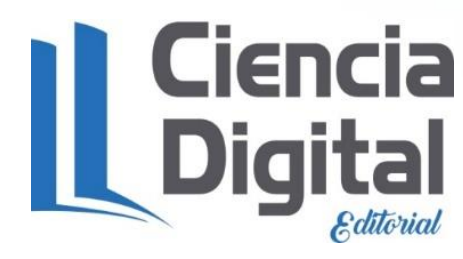


El artículo que se publica es de exclusiva responsabilidad de los autores y no necesariamente reflejan el pensamiento de la Revista Conciencia Digital.

\section{Ciencia \\ LDigital}

El artículo queda en propiedad de la revista y, por tanto, su publicación parcial y/o total en otro medio tiene que ser autorizado por el director de la Revista Conciencia Digital.
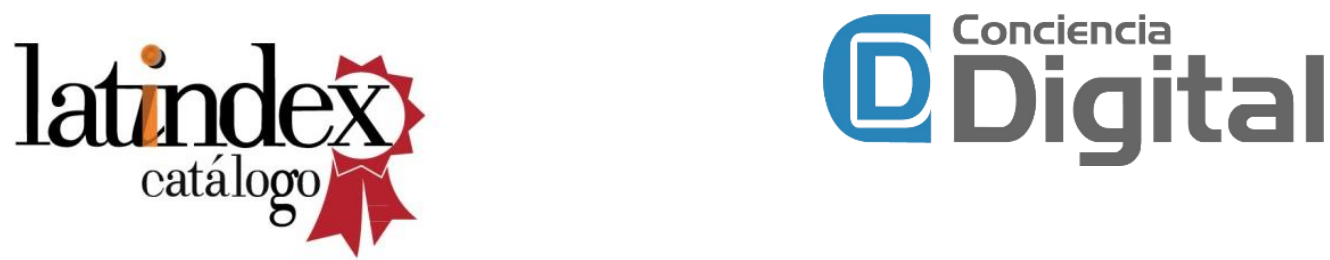

Indexaciones

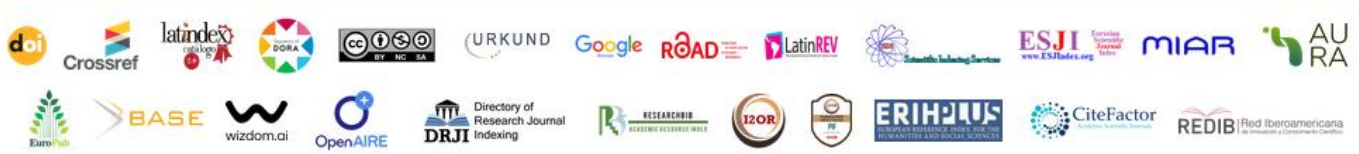

